SARS-CoV-2 B.1.1.7 variant-Texas, January 2021. Morb Mortal Wkly Rep 2021;70:348-349.

5. US COVID-19 cases caused by variants. Centers for Disease Control and Prevention website. https://www.cdc.gov/coronavirus/2019-ncov/ transmission/variant-cases.html. Published 2021. Accessed March 28, 2021.

6. Babiker A, Marvil CE, Waggoner JJ, Collins MH, Piantadosi A. The importance and challenges of identifying SARS-CoV-2 reinfections. J Clin Microbiol 59:e02769-20.
7. Tillett RL, Sevinsky JR, Hartley PD, et al. Genomic evidence for reinfection with SARS-CoV-2: a case study. Lancet Infect Dis 2021;21:52-58.

8. Visseaux B, Le Hingrat Q, Collin G, et al. Evaluation of the RealStar SARSCoV-2 RT-PCR kit RUO performances and limit of detection. J Clin Virol 2020.

9. Paden CR, Tao Y, Queen K, et al. Rapid, sensitive, full-genome sequencing of severe acute respiratory syndrome coronavirus 2. Emerg Infect Dis 2020;26:2401-2405.

\title{
CDC mask recommendations and guideline development: Missing pieces
}

\author{
Sajith Matthews MD, FACP \\ Department of Internal Medicine, Division of General Medicine, Wayne State University Detroit, Michigan
}

To the Editor-The Center for Disease Control (CDC) guidelines ${ }^{1}$ for masks would benefit from an appraisal by the standardized instrument of AGREE $\mathrm{II}^{2}$ (Appraisal of Guidelines for Research \& Evaluation) because questions in the domains of stakeholder involvement and rigor of development remain unanswered. AGREE II assesses the quality of a guideline in the domains of scope and purpose, stakeholder involvement, rigor of development, applicability, editorial independence, and clarity of presentation, with 2-4 independent appraisals that require an average $>70 \%$ to be considered a high-quality guideline. When evaluating the recent updates to the CDC mask guidelines, the AGREE II instrument may provide clarity to the mask guideline development process, its strengths, and its deficiencies.

The rigor of development for mask guidelines has important components that are unreported, specifically (1) the criteria for selecting the evidence, (2) the explicit link between the recommendations and supporting evidence, and (3) the consideration of health benefits, side effects, and risks. The criteria for selecting the evidence is unclear, especially with observational studies rather than randomized control trials (RCTs) being used to assess mask efficacy. The former is typically useful for risk assessment and the latter for efficacy of an intervention. ${ }^{3}$ Meta-analyses of observational studies ${ }^{4}$ have failed to demonstrate a large enough treatment effect of masks $(\mathrm{RR}<0.50)$ to mark up the rating of the quality of evidence to replace RCTs. ${ }^{5,6}$ On the contrary, the RCTs for mask use have shown little efficacy in preventing the transmission of respiratory infections. ${ }^{7}$ The recent DANMASK 19 trial, assessing universal masking for preventive effect, also showed that the effectiveness of masks was negligible in preventing the transmission of severe acute respiratory syndrome coronavirus 2 (SARS-CoV-2) when other nonpharmaceutical interventions (NPI) were in place. ${ }^{8}$ Conventionally, the more restrictive the guidance (ie, universal masking), the more certain the guideline developers are of its correctness. ${ }^{9}$

The explicit link between the recommendations and supporting evidence is missing in the recommendation for placing a cloth mask over a surgical procedure mask (double masking). The

Author for correspondence: Sajith Matthews, E-mail: smatthew@med.wayne.edu Cite this article: Matthews S. (2022). CDC mask recommendations and guideline development: Missing pieces. Infection Control \& Hospital Epidemiology, 43: 1090-1091, https://doi.org/10.1017/ice.2021.197 evidence is based on an experiment demonstrating that a 3-ply medical procedure mask covered by a 3-ply cloth cotton mask blocked $92.5 \%$ of potassium chloride particles on a pliable elastomeric head form used to simulate a person coughing and producing aerosols from a mouthpiece. ${ }^{1}$ It is crucial that the confidence rests in direct evidence from similar human populations and outcomes to those targeted by the guideline rather than preclinical studies, which are intended to be exploratory and hypothesis generating. Although translational medicine acts as a bridge, its translatability from preclinical science to human application is often irreproducible. ${ }^{10}$ Therefore, the leap from basic science research (T0) to translation to the community (T4) without assessing safety and proof of efficacy would be unprecedented.

A balanced assessment of the benefits and harms of universal masking (and double masking) is needed. Studies on the benefits and harms of wearing medical masks are limited, increased dyspnea and work of breathing, hypoxemia, hypercapnia and headaches have been reported. ${ }^{11,12}$ Therefore, claim that universal mask use is a relatively benign measure ${ }^{13}$ is imprecise.

Pertaining to stakeholder involvement, whether views and preferences of the target population (public) have been sought remains unreported. The impact of mask use on the psychological needs (autonomy, competence, and relatedness) has been well documented $^{14}$; therefore, including public's views in guideline development would be essential to the process. This is even more relevant with double masking because a negative attitude of masks due to psychological reactance and perceived ineffectiveness has been well described. ${ }^{15}$ Whether the guideline development group included individuals from psychiatry remains unclear.

Although many of the CDC mask guidelines were interim guidelines due to the urgency of the pandemic, applying the slower, more robust guideline development process would be advisable. Providing the missing pieces in the domains of stakeholder involvement and rigor of development for the CDC recommendations would make the guidelines more comprehensive. The question of whether AGREE II is an appropriate appraising instrument to use during the pandemic is reasonable. However, it is the only tool that has been validated internationally, being cited in $>650$ publications. ${ }^{16}$ AGREE II contains the necessary domains to assess methodological rigor, transparency of development and the overall quality of the mask guidelines, providing the much-needed 
veracity for the public and for health officials. Alternatively, failure to adequately address these domains may erode of the public's trust in public health recommendations.

Acknowledgments. The author's affiliation center belongs to the Integrative Biosciences Center, Wayne State University, Detroit, Michigan. The funders had no role in the decision to publish or preparation of the manuscript.

Financial support. No financial support was provided relevant to this article.

Conflicts of interest. The author reports no conflicts of interest relevant to this article

\section{References}

1. Brooks JT, Beezhold DH, Noti JD, et al. Maximizing fit for cloth and medical procedure masks to improve performance and reduce SARS-CoV-2 transmission and exposure, 2021. Morb Mortal Wkly Rep 2021;70:254-257.

2. Brouwers MC, Kerkvliet K, Spithoff K. The AGREE reporting checklist: a tool to improve reporting of clinical practice guidelines. BMJ 2016;352: i1152.

3. Shekelle PG, Woolf SH, Eccles M, Grimshaw J. Clinical guidelines: developing guidelines. BMJ 1999;318:593-596.

4. Chu DK, Akl EA, Duda S, Solo K, Yaacoub S, Schünemann HJ; COVID-19 Systematic Urgent Review Group Effort (SURGE) study authors. Physical distancing, face masks, and eye protection to prevent person-to-person transmission of SARS-CoV-2 and COVID-19: a systematic review and meta-analysis. Lancet 2020;395:1973-1987.

5. Murad MH. Clinical practice guidelines: a primer on development and dissemination. Mayo Clin Proc 2017;92:423-433.

6. Frieden TR, Cash-Goldwasser S. Of masks and methods. Ann Intern Med 2021. doi: 10.7326/M20-7499.

7. Nanda A, Hung I, Kwong A, Man VC, Roy P, Davies L, Douek M. Efficacy of surgical masks or cloth masks in the prevention of viral transmission: systematic review, meta-analysis, and proposal for future trial. J Evid Based Med 2021. doi: 10.1111/jebm.12424.

8. Bundgaard H, Bundgaard JS, Raaschou-Pedersen DET, et al. Effectiveness of adding a mask recommendation to other public health measures to prevent SARS-CoV-2 infection in Danish mask wearers: a randomized controlled trial. Ann Intern Med 2020:M20-6817.

9. American Academy of Pediatrics Steering Committee on Quality Improvement and Management. Classifying recommendations for clinical practice guidelines. Pediatrics 2004;114:874-877.

10. Ioannidis JP. Why most clinical research is not useful. PLoS Med 2016;13: e1002049.

11. Does the prolonged use of face masks by HCW interfere with the respiratory system by inducing oxidative stress and blood oxygen/carbon dioxide imbalance? National Health Library and Knowledge Service website. https:// hselibrary.ie/does-the-prolonged-use-of-face-masks-by-hcws-interfere-withthe-respiratory-system-by-inducing-oxidative-stress-and-blood-oxygen-carbondioxide-imbalance/. Published December10, 2020. Accessed February 18, 2021.

12. Rosner E. Adverse effects of prolonged mask use among healthcare professionals during COVID-19. J Infect Dis Epidemiol 2020;6:130.

13. Samannan R, Holt G, Calderon-Candelario R, et al. Effect of face masks on gas exchange in healthy persons and patients with chronic obstructive pulmonary disease. Ann Am Thorac Soc 2021;18:541-544.

14. Scheid JL, Lupien SP, Ford GS, West SL. Commentary: physiological and psychological impact of face mask usage during the COVID-19 pandemic. Int J Environ Res Public Health 2020;17:6655.

15. Taylor S, Asmundson GJG. Negative attitudes about facemasks during the COVID-19 pandemic: the dual importance of perceived ineffectiveness and psychological reactance. PLoS One 2021;16(2):e0246317.

16. Brouwers MC, Kho ME, Browman GP, et al. AGREE Next Steps Consortium. AGREE II: advancing guideline development, reporting and evaluation in health care. CMAJ 2010;182:E839-E842.

\title{
"Original antigenic sin": A potential threat beyond the development of booster vaccination against novel SARS-CoV-2 variants
}

\author{
Maryam Noori $\mathrm{MD}^{1}$, Seyed Aria Nejadghaderi $\mathrm{MD}^{2,3}$ and Nima Rezaei MD, PhD ${ }^{4,5,6}$ (1) \\ ${ }^{1}$ Student Research Committee, School of Medicine, Iran University of Medical Sciences, Tehran, Iran, ${ }^{2}$ Systematic Review and Meta-analysis Expert Group \\ (SRMEG), Universal Scientific Education and Research Network (USERN), Tehran, Iran, ${ }^{3}$ School of Medicine, Shahid Beheshti University of Medical Sciences, \\ Tehran, Iran, ${ }^{4}$ Research Center for Immunodeficiencies, Children's Medical Center, Tehran University of Medical Sciences, Tehran, Iran, ${ }^{5}$ Department of \\ Immunology, School of Medicine, Tehran University of Medical Sciences, Tehran, Iran and ${ }^{6}$ Network of Immunity in Infection, Malignancy and Autoimmunity \\ (NIIMA), Universal Scientific Education and Research Network (USERN), Tehran, Iran
}

To the Editor-Recently, concern has increased over the emergence of novel severe acute respiratory syndrome coronavirus 2 (SARS-CoV-2) variants, which are spreading rapidly across the globe. These variants of concern (B.1.1.7, B.1.351, P.1, and B.1.427/429) have been initially reported in the United Kingdom, South Africa, Brazil, and California, respectively. ${ }^{1}$ All of the currently available vaccines that have received emergency use authorization, such as Johnson \& Johnson, Moderna, and Pfizer/BioNTech, are based on the Wuhan-originated virus.

Author for correspondence: Prof Nima Rezaei, E-mail: rezaei_nima@tums.ac.ir or rezaei_nima@yahoo.com

Cite this article: Noori M, Nejadghaderi SA, and Rezaei N. (2022). “Original antigenic sin": A potential threat beyond the development of booster vaccination against novel SARS-CoV-2 variants. Infection Control \& Hospital Epidemiology, 43: 1091-1092, https://doi.org/10.1017/ice.2021.199
Regarding the novel variants, the accumulation of multiple mutations in the spike protein, which is the target for neutralizing antibodies, has challenged the efficacy of these vaccines. Several previous laboratory-based studies have reported that the neutralizing activity of sera obtained from individuals who were vaccinated is lower against novel SARS-CoV-2 variants, ${ }^{2-5}$ highlighting the need for developing a booster vaccination containing new mutations of the virus.

A phenomenon called "original antigenic sin" (OAS) was firstly proposed by Francis ${ }^{6}$ in 1960. This phenomenon occurs in the second exposure of the immune system to a similar pathogen to which it has previously been exposed. In this situation, the immune system progresses to the memory response, generating crossreactive antibodies that may not be effective against the new pathogen. ${ }^{7}$ In addition, it has been speculated that overproduction 